\title{
An Investigation into the Integrity of Fit of Provisional Crowns made over a Dental Implant Analogue Using Two Current Proprietary Provisional Crown Materials Compared to a Proprietary 'Snap 0n' Provisional Core
}

\author{
Georgios Georgakis ${ }^{1 *}$, Philip D Taylor ${ }^{1}$ and Jason ${ }^{2}$ \\ ${ }^{1}$ Department of Prosthodontics, Bart's and The London School of Medicine and Dentistry, Lefkada, Greece \\ ${ }^{2}$ Department of Adult Oral Health, Bart's and The London School of Medicine and Dentistry, Lefkada, Greece
}

Received: September 08, 2014; Accepted: October 31, 2014; Published: November 12, 2014

*Corresponding author: Georgios Georgakis, Department of Prosthodontics, Bart's and The London School of Medicine and Dentistry, Lordou Byrona 41, 31100 Lefkada, Greece, Tel: +30-26-45-02-15-60; E-mail: georgakisgeorge@yahoo.gr

\begin{abstract}
The aim of this study was to investigate the marginal accuracy of three currently used methods of direct provisional crown construction for the accuracy of marginal fit during in vitro fabrication. Three groups of provisional crowns were investigated using a modified McLean and von Fraunhofer technique. Proprietary provisional crown coping modified to tooth shape with a Bis-acryl temporary material was compared to Bis-GMA and isobutyl methacrylate resin provisional crowns with modified margins using a light cured flowable composite and 'bead on' isobutyl methacrylate respectively. Data was analyzed with the Mann Whitney test $(a=0.05)$. Reliability was determined using the Bland Altman test. Bis-GMA acryl resin composite relined with flowable composite produced significantly better fitting restorations compared to the two other groups.
\end{abstract}

Keywords: Provisional crowns; Marginal fit; Bis-acryl temporary; Temporary materials; Composite; Crown coping

\section{Introduction}

The importance of provisional restorations in both conventional and implant prosthodontics, is well documented in the dental literature [1] and the requirements for satisfactory provisional restorations differ only slightly from the definitive treatment they precede. The rationale for provisional coverage of teeth and implant abutments identifies multiple areas of concern including aesthetics, comfort, speech, function, periodontal health, maxilla-mandibular relationships, and continued evaluation of the fixed prosthodontic treatment plan [2-4]. Biologically acceptable fixed prosthodontic treatments demands that prepared teeth should be protected and stabilized with provisional restorations that resemble the form and function of the planned definitive treatments. Around implant abutments, they can promote guided tissue healing by providing a matrix for the surrounding gingival tissue [5].
Provisional restorations are also useful for diagnostic purposes. The functional, occlusal, and aesthetic parameters can be assessed before the completion of definitive prostheses [5]. A provisional restoration can provide a template for defining tooth contour, shade, emergence profile, proximal contacts of the definitive restoration, contributing also to optimal patientdentist communication. The patient can really visualize the treatment outcome and understand the possible limitations [6]. Finally, the occlusal scheme can be evaluated [6], as well as the potential consequences from an alteration in the occlusal vertical dimension [1].

Historically, autopolymerizing Poly-Methyl Methacrylate (PMMA) and Poly-Ethyl Methacrylate (PEMA) resins have been the materials of choice for the construction of provisional crowns and bridges. However, Bis-GMA acryl resin composites have become a popular alternative choice due to their ease of use. Research has shown Bis-GMA acryl resin composite to provide some advantages in physical properties including low polymerization shrinkage [7] and good marginal adaptation [8-10] low exothermic reaction [11,12] minimal pupil and soft tissue irritation [13], good surface hardness [14], and increased color stability [2,12].

Until recently, alterations for repairs and addition of Bis-GMA acryl resin to composite material was difficult, even though they are compatible with other composite materials [1,2]. Studies have found an $85 \%$ decrease in transverse strength after repair of a Bis-GMA acryl resin provisional material [15]. It has been suggested on the same studies that it might be more advantageous to make new provisional restorations than repair restorations made from Bis-GMA acryl resin provisional materials. However, clinical experience has shown that flowable composite resins can be used to repair Bis-GMA acryl resin provisional restorations with ease and success [16]. 
Numerous studies appear in the dental literature comparing the marginal fit of provisional restorations made from different types of materials [8-10,17] and different techniques [18-21] as well as the effect of environment's temperature [22] and storage conditions [23]. Dental implant companies, on the other hand, have all tried to make the temporization phase of treatment more predictable and many have produced stock templates for their own implant systems. The accuracy of these templates when modified to tooth shape has not been documented in the literature. The purpose of this investigation is to compare the accuracy of fit of three manufacturing methods under the test conditions in vitro and investigate the null hypothesis that there is no difference in the accuracy of fit of the three manufacturing methods under the test conditions in vitro.

\section{Materials and Methods}

In this investigation provisional single crowns were made over an implant abutment (Solid abutment; ITI Straumann, Crawley, W.Sussex, UK) 7 mm high code number 048.927 using 3 different methods. The accuracy of fit of provisional crowns made from isobutyl methacrylate acrylic resin (Trim II; Skokie III) with their margins refined with the 'bead on' or 'paint on' technique were compared with those made from Bis-GMA acryl resin composite (Protemp 3 Garant; 3M ESPE, Loughborough, Leicestershire, UK) relined with flowable composite, and those produced using the implant abutment temporary coping (ITI Straumann, W.Sussex, UK) code number 048.655 modified to tooth shape with Bis-GMA acryl resin composite as above but no marginal modification.

\section{Analogue preparation}

A standard ITI Straumann $4.8 \mathrm{~mm}$ regular neck $12 \mathrm{~mm}$ length implant fixture (ITI Straumann, W.Sussex, UK) code number 033.253S, was embedded into a brass cylinder and a ITI Straumann Solid abutment $(7 \mathrm{~mm})$ torque into position leaving a sloping shoulder margin (Figure 1a). Two location notches were prepared on the brass cylinder to allow reproducibility. A canine tooth was waxed up over the abutment to mimic a clinical situation (Figure 1b). A cylinder using light cured composite tray material (Composite tray material; Bracon Ltd, Etchingham, East Sussex, UK) was made to fit accurately around the brass casting which included two lugs on its internal surface (Figure 1c).

Silicone putty (ExtrudeXP; Kerr Ltd, Peterborough, UK) was used to create a matrix which was used for the construction of the provisional crowns. In the surface of the putty matrix a notch was created with a number 10 scalpel, in order to allowthe excess of provisional material to be vented and secure the complete seating of the matrix over the analogue. Twenty five provisional crowns were made in each of the three groups tested.

\section{Sample preparations}

\section{Group 1: Isobutyl methacrylate}

Isobutyl methacrylate provisional material (Trim II; H.J. Bosworth) was mixed according to the manufacturer's instructions. A plastic pipette was used to place $7 \mathrm{ml}$ of liquid

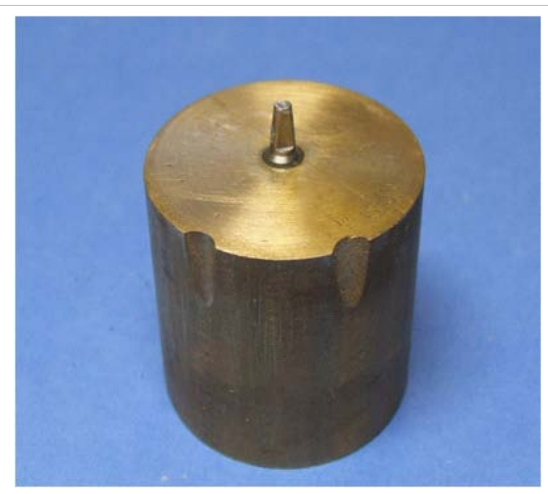

Figure 1a: Implant fixture and ITI Straumann Solid abutment embedded into brass cylinder also showing 2 location notches on brass cylinder to allow reproducibility.

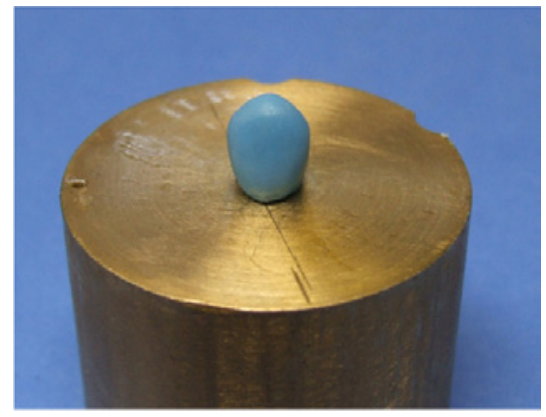

Figure 1b: Canine tooth waxed up over abutment.

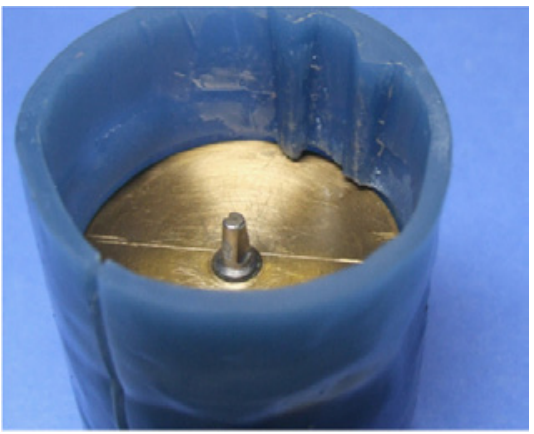

Figure 1c: Cylinder from light cured composite tray material made to fit accurately around brass casting which included 2 lungs on its internal surface.

into a plastic mixing cup and $12.5 \mathrm{~g}$ of powder were added. The material was mixed for 1 minute with a metal spatula. The putty matrix was filled with the provisional material and after another minute, when the material reached the dough consistency, the putty matrix was seated over the abutment. The material was left for 3 minutes to ensure polymerization before the putty matrix was carefully removed from the analogue without touching the margins and was left to completely cure on the bench for 15 minutes according to the manufacturer's instructions.

The excess material at the margin was trimmed with polishing discs (Soflex; 3M ESPE) at x2.5 magnification (Rose 
Micro Solutions; x2.5 loupes). The crown margin was re-defined using the 'bead on' technique as described by Zwetchkenbaum [17]. The fresh provisional material was left to polymerize for 15 minutes. Once cured the acrylic resin provisional crown margin was further trimmed and then polished under x2.5 magnification loupes with medium, fine and super fine polishing discs (Soflex; 3M ESPE). A total of 25 provisional crowns were made in this manner with Trim II.

\section{Group 2: Bis-GMA acryl resin composite}

The Bis-GMA acryl resin composite (Protemp 3 Garant; 3M ESPE) provisional material is supplied in the Garant Dispenser which was used for mixing of the two pastes and its subsequent application of the provisional material directly into the putty matrix. This was then seated over the analogue and left to set for 2 minutes. The provisional crown was carefully removed from the analogue without touching the margins and was left to completely cure on the bench for 5 minutes according to the manufacturer's instructions and then trimmed in the same manner as described in Group 1 except for this group the relining procedure involved the application of bonding agent (Optibond Solo plus; Kerr) to the restoration's margin which was light cured for 15 seconds. The provisional crown was then relined with flowable composite (Protemp Add On; 3M ESPE) and re-positioned. This in turn was light cured (mesially, distally, buccally, and lingually) for 15 seconds on each surface after placement back on the analogue. A total of 25 provisional crowns were made in this manner with Protemp 3 Garant.

Group 3: Modified stock templates provided from the implant manufacturers

The ITI Straumann temporary coping for regular neck Solid abutment was used for the preparation of the third group of this investigation. According to the manufacturer the temporary coping is made from PMMA and has a height of $8.5 \mathrm{~mm}$. The surface of the temporary coping is serrated to provide mechanical retention for the provisional material used over it. A wide range of provisional materials can be used over it for provisionalization.

After the ITI Straumann temporary coping was clipped in place over the fixture head the putty matrix was filled with BisGMA acryl resin composite (Protemp 3 Garant; 3M ESPE), and fitted it. The procedure for polishing was repeated for this group except that no reline or margination procedure was used. A total of 25 provisional crowns were made in this manner with the temporary copping.

\section{Sample measuring}

An in vivo technique for the estimation of cement thickness on definitive fixed restorations has been proposed by McLean and von Fraunhofer [24]. A modification of this technique was used in this experiment by using silicone impression materials. The fit surface of the provisional crowns was filled with low viscosity polyvinyl siloxane impression material (Extrude; Kerr) and the restoration was fitted over the Solid ITI Straumann abutment to mimic the cement lute (Figure 2a) and left to set for 7 minutes. The provisional crown was then removed from the implant abutment having the low viscosity polyvinyl siloxane on the fit surface (Figure 2b). The remaining space was filled with medium body polyvinyl siloxane (Extrude; Kerr) impression material (Figure 2c) and left to set for further 7 minutes.

The light and medium body silicone was removed from the fit-surface of the provisional crowns and covered with low viscosity polyvinyl siloxane impression material of another company (Aquasil XLV Ultra; Dentsply, Weybridge, Surrey, UK) which has a different colour than the one used to record the fit surface of the provisional crown. The low viscosity impression material was left to set for a further 7 minutes and after setting sectioned down the center with a number 10 scalpel. The sections were cut from the mesial to distal aspect as proposed by McLean and von Fraunhofer [24].

An Olympus BX60 optical microscope (Olympus Ltd, Watford, Hertfordshire, UK) was used to measure the thickness of the low viscosity Extrudeimpression material on all the sections of the rubber impression samples under x50 magnification. Seven measurements were made in total on each rubber impression sample (Figure $2 \mathrm{~d}$ ). The points of measurement for each sample are presented schematically in (Figure 2e).

Measurement A: The first measurement was made on the most external point of the margin on the mesial site (left hand side) of the rubber impression sample.

Measurement B: The second measurement was made $2 \mathrm{~mm}$

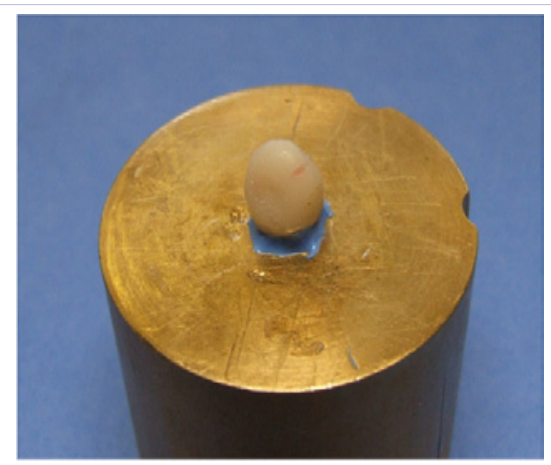

Figure 2a: The fit surface of the provisional crowns was filled with light body polyvinyl siloxane impression material and the restoration was fitted over the Solid ITI Straumann abutment to mimic the cement lute.

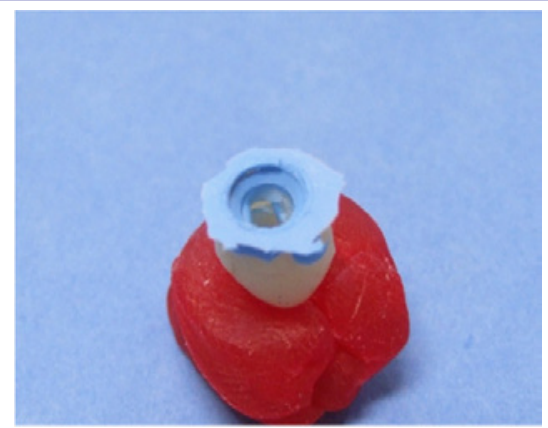

Figure 2b: The provisional crown was removed from the implant abutment having the light body polyvinylsiloxane on the fit surface. 


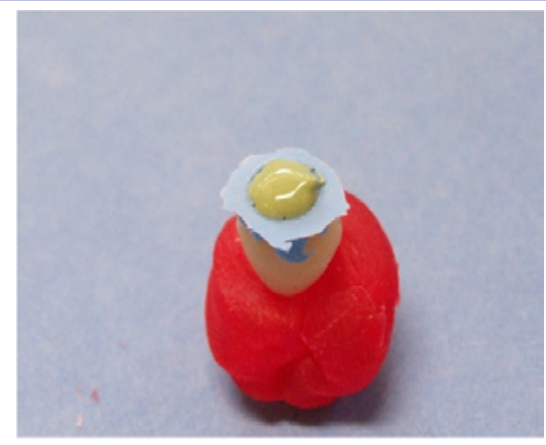

Figure 2c: The remaining space was filled with medium body polyvinyl siloxane impression material.

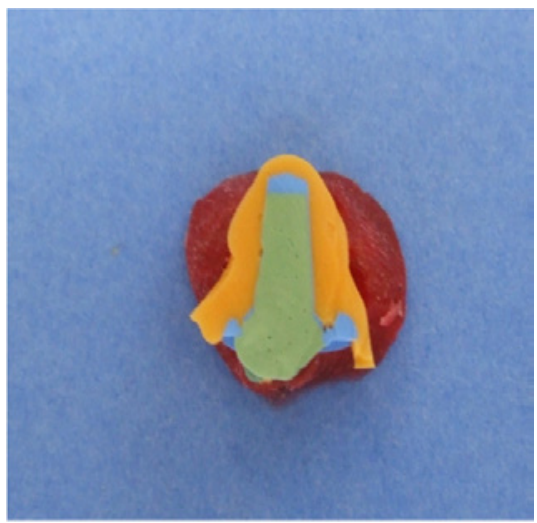

Figure 2d: Sectioned.

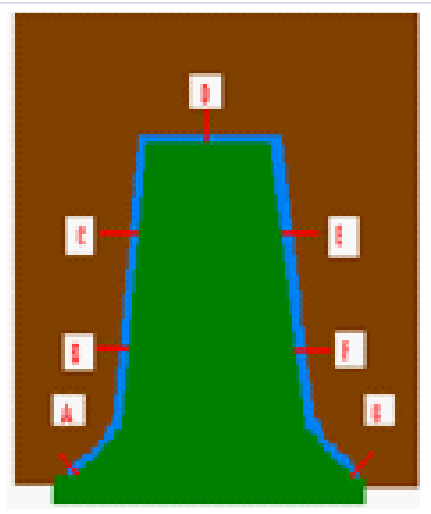

Figure 2e: Schematic presentation of measurement points.

occlusally of the restoration's margin on the mesial axial wall of the rubber impression sample.

Measurement C: The third measurement was made $4 \mathrm{~mm}$ occlusally from the restoration's mesial margin on the mesial axial wall of the rubber impression sample.

Measurement D: The fourth measurement was made at the middle of the occlusal surface of the rubber impression sample.

Measurement E: The fifth measurement was made $4 \mathrm{~mm}$ occlusally of the restoration's distal margin on the distal axial wall of the rubber impression sample.
Measurement F: The sixth measurement was made $2 \mathrm{~mm}$ occlusally of the restoration's distal margin on the distal axial of the rubber impression sample.

Measurement G: The seventh measurement was made on the most external point of the margin on the distal site (right hand side) of the rubber impression sample.

A total of 75 measurements were made at each point in all 3 groups tested.

\section{Statistical analysis}

Data analysis included descriptive and analytical statistics using the Statistical Package for Social Science (SPSS Inc version 13.0.1). All data in the present study were quantitative; therefore descriptive statistics included the presentation of measurement's median and range.

Analysis of the data in the study involved two parts. The first part assessed the reliability of the measures used in this study. The method described by Bland and Altman [25], was used to demonstrate the precision of measurements taken.

The second part tested the hypotheses of the study, which requires analytical statistics. Non- parametric tests were selected, because the sample's size was small, thus the assumptions for parametric tests were not met. The Mann-Whitney test was chosen to compare the differences in the calculated distances of the samples produced by the three provisional materials under investigation. The level of statistical significance was set at 0.05 .

\section{Results}

The first part assessed the reliability of the measures used in this study using the method described by Bland and Altman [25]. Repeated measurements were taken from seven randomly selected samples of each group and their agreement with the original measurements taken in the study was tested. All measurements demonstrated very good agreement; the majority of them differentiate with the original measurements by less than $1 \mu \mathrm{m}$ in most cases.

Descriptive statistics are presented at (Table 1). Analytical statistics resulted at significant differences between the isobutyl methacrylate and Bis-GMA acryl resin composite groups in almost all points of measurement. Results on the accuracy of fit indicate that Bis-GMA acryl resin composite produced better fitting provisional crowns than Isobutyl methacrylate. Only at points $\mathrm{E}$ and $\mathrm{F}$ the differences was not of statistical significance ( $p$ $>0.05$ ) although Bis-GMA acryl resin composite exhibited better fitting restorations than Isobutyl methacrylate. The results of this comparison are presented at (Table 2).

Overall, highly statistical significant differences were found between the Isobutyl methacrylate and the ITI Straumann temporary coping modified on tooth form with Bis-GMA acryl resin composite resin groups in all points of measurement. Results on the accuracy of fit indicate that Isobutyl methacrylate produced the best fitting provisional crowns over the ITI Straumann temporary coping modified to tooth form with BisGMA acryl resin composite. Detailed comparison of discrepancies 
Table 1: Mean values and standard deviation in all points of measurement for all groups.

\begin{tabular}{|c|c|c|c|c|}
\hline Material & $\begin{array}{c}\text { Point of } \\
\text { measurement }\end{array}$ & $\mathbf{N}$ & Mean & $\begin{array}{l}\text { Standard } \\
\text { Deviation }\end{array}$ \\
\hline \multirow{7}{*}{ 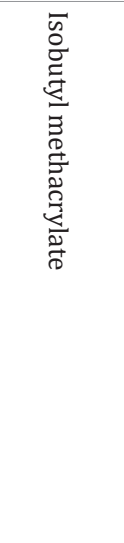 } & Measurement A & 25 & 83.61 & 12.22 \\
\hline & Measurement B & 25 & 29.30 & 20.00 \\
\hline & Measurement C & 25 & 34.36 & 21.99 \\
\hline & Measurement D & 25 & 184.52 & 97.28 \\
\hline & Measurement E & 25 & 19.91 & 19.75 \\
\hline & Measurement F & 25 & 19.81 & 15.71 \\
\hline & Measurement G & 25 & 84.46 & 14.85 \\
\hline \multirow{7}{*}{ 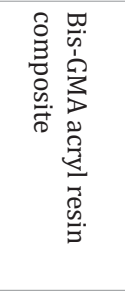 } & Measurement A & 25 & 69.97 & 16.21 \\
\hline & Measurement B & 25 & 17.49 & 11.37 \\
\hline & Measurement C & 25 & 14.67 & 10.52 \\
\hline & Measurement D & 25 & 35.94 & 18.68 \\
\hline & Measurement E & 25 & 15.83 & 7.82 \\
\hline & Measurement F & 25 & 16.90 & 9.45 \\
\hline & Measurement G & 25 & 71.12 & 18.07 \\
\hline \multirow{7}{*}{ 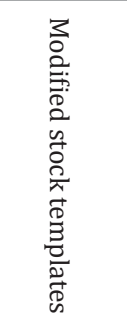 } & Measurement A & 25 & 203.00 & 31.67 \\
\hline & Measurement B & 25 & 72.30 & 22.77 \\
\hline & Measurement C & 25 & 74.72 & 18.33 \\
\hline & Measurement D & 25 & 392.15 & 126.36 \\
\hline & Measurement E & 25 & 63.51 & 22.71 \\
\hline & Measurement F & 25 & 61.43 & 23.70 \\
\hline & Measurement G & 25 & 240.70 & 67.91 \\
\hline
\end{tabular}

Table 2: Statistical comparison between Group 1 and 2 using MannWhitney test.

\begin{tabular}{|c|c|c|c|}
\hline Point of measurement & Group & Mean Rank & $P$ value \\
\hline \multirow{2}{*}{ Measurement A } & Group 1 & 31.50 & \multirow{2}{*}{0.004} \\
\hline & Group 2 & 19.50 & \\
\hline \multirow{2}{*}{ Measurement B } & Group 1 & 30.80 & \multirow{2}{*}{0.010} \\
\hline & Group 2 & 20.20 & \\
\hline \multirow{2}{*}{ Measurement C } & Group 1 & 32.48 & \multirow{2}{*}{0.001} \\
\hline & Group 2 & 18.52 & \\
\hline \multirow{2}{*}{ Measurement D } & Group 1 & 38.00 & \multirow{2}{*}{0.000} \\
\hline & Group 2 & 13.00 & \\
\hline \multirow{2}{*}{ Measurement E } & Group 1 & 25.08 & \multirow{2}{*}{0.839} \\
\hline & Group 2 & 25.92 & \\
\hline \multirow{2}{*}{ Measurement G } & Group 1 & 26.20 & \multirow{2}{*}{0.734} \\
\hline & Group 2 & 24.80 & \\
\hline \multirow{2}{*}{ Measurement F } & Group 1 & 31.28 & \multirow{2}{*}{0.005} \\
\hline & Group 2 & 19.72 & \\
\hline
\end{tabular}

produced by the two materials under investigation is presented at (Table 3).

\section{Reliability of clinical measurements in the study}

The reliability of the measures used in this study was assessed using the method described by Bland and Altman (1986) [25]. Repeated measurements were taken from seven randomly selected samples of each group and their agreement with the original measurements taken in the study was tested. All measurements demonstrated very good agreement; the majority of them differentiate with the original measurements by less than $1 \mu \mathrm{m}$ in most cases.

\section{Discussion}

In this in vitro investigation, provisional restorations were made by direct method. The restorations were removed from the analogue at the onset of the initial polymerization, according to the material manufacturer's advice, and allowed to complete polymerization off the analogue at room temperature. Removal of provisional restorations before significant curing can result in distortion especially in critical areas such as restorations margins [9]. The removal technique in which restorations are left on the bench to complete polymerization also results in increased marginal discrepancies than other techniques that allow curing of the restorations on the prepared natural tooth or implant abutment [19] or the indirect fabrication of provisional restorations $[19,20]$. Relining techniques with or without venting of the restoration are proven to significantly improve marginal adaptation $[17,19,21]$.

All groups in this experiment exhibited better fit in the axial walls than at the restorations margins or under the occlusal surfaces. This observation can be explained from the fact that polymerization shrinkage occurs towards the bulk of the material forcing the material to shrink away from the restoration's margins. The increased space under the restoration's occlusal

Table 3: Statistical comparison between Group 1 and 3 using MannWhitney test.

\begin{tabular}{|c|c|c|c|}
\hline Point of measurement & Group & Mean Rank & $P$ value \\
\hline \multirow{2}{*}{ Measurement A } & Group 1 & 13.00 & \multirow{2}{*}{0.000} \\
\hline & Group 3 & 38.00 & \\
\hline \multirow{2}{*}{ Measurement B } & Group 1 & 15.24 & \multirow{2}{*}{0.000} \\
\hline & Group 3 & 35.76 & \\
\hline \multirow{2}{*}{ Measurement C } & Group 1 & 15.48 & \multirow{2}{*}{0.000} \\
\hline & Group 3 & 35.52 & \\
\hline \multirow{2}{*}{ Measurement D } & Group 1 & 15.40 & \multirow{2}{*}{0.000} \\
\hline & Group 3 & 35.60 & \\
\hline \multirow{2}{*}{ Measurement E } & Group 1 & 14.68 & \multirow{2}{*}{0.000} \\
\hline & Group 3 & 36.32 & \\
\hline \multirow{2}{*}{ Measurement G } & Group 1 & 14.72 & \multirow{2}{*}{0.000} \\
\hline & Group 3 & 36.28 & \\
\hline Measurement F & Group 1 & 13.00 & 0.000 \\
\hline
\end{tabular}


surfaces for Groups 1 and 2 might be the result of incomplete seating of the restorations on the analogue due to the close fit of the restorations in the axial walls.

For Group 3 the significantly increased space under the occlusal surface, which reached the $585 \mu \mathrm{m}$ in one restoration, can be explained by the fact that the temporary coping is a hollow cylinder $1.5 \mathrm{~mm}$ higher than the ITI Straumann Solid abutment and that space was not completely filled with the provisional material during its modification in tooth form. Even the use of low viscosity silicone impression material to record the space between the provisional crowns and the analogue used in this in vitro investigation might have caused incomplete seating of the crowns resulting in increased marginal discrepancies and increased space under the occlusal surfaces of the restorations. The use of cement lute in clinical practice can have the same effect on the restorations, thus the technique employed can result in a fit similar to that produced in clinical practice [24].

McLean and von Fraunhofer [24] reported that the use of polyether rubber to mimic the cement lute can reproduce films as thin as $10 \mu \mathrm{m}$ quite accurately. It was observed in our investigation that the use of modern polyvinylsiloxane impression materials can reproduce film thickness less than $10 \mu \mathrm{m}$. Films as thin as 5 $\mu \mathrm{m}$ were recorded on the axial walls of provisional restorations made from isobutyl methacrylate and Bis-GMA acryl resin composite. As the ISO standard for acceptance as a cement lute is $25 \mu \mathrm{m}$ such a close adaptation of the provisional restorations to the axial walls of the analogue may cause incomplete sitting of the restorations during clinical practice.

A number of studies have compared acrylic methacrylate based provisional materials with Bis-GMA acryl resin composite materials reporting the superiority of Bis-GMA acryl resin composite in terms of marginal fit, but the restoration's fit on the axial walls and under the restoration's occlusal surfaces has not been evaluated. Also, in our knowledge there are no studies comparing those materials with temporary copings provided from implant manufacturers to simplify provisionalization phase over implant abutments.

A study which compared 6 different provisional materials including those used in our study reported similar findings in terms of marginal integrity [12]. The mean marginal discrepancies reported were $110 \mu \mathrm{m}$ for Isobutyl methacrylate and $95 \mu \mathrm{m}$ for Bis-GMA acryl resin composite. No information is given in that study whether the restorations were left to complete polymerization over the analogue or whether they were removed. No margination technique was performed in that study which may explain the small differences with our study.

The materials used on our experiment were also compared in terms of marginal adaptation in another study which concluded that both materials result in mean marginal discrepancies of 60 $\mu \mathrm{m}$ [18]. In that study the restorations were only removed once from the analogue for a short period of time, and then they were reseated on the analogue and polymerization completed by immersing them in a water bath at $37^{\circ} \mathrm{C}$. No reline or margination technique was performed.
The same methodology was used in a number of other studies. Restorations were placed in a water bath at $37^{\circ} \mathrm{C}$ to complete polymerization and a spring loading holding device was used to secure the interim crowns over the analogue during measurements were performed with a measuring microscope. Tjan et al. [9] reported marginal discrepancies of $40.1 \mu \mathrm{m}$ for BisGMA acryl resin composite and $23.6 \mu \mathrm{m}$ for isobutyl methacrylate. The same group of investigators in a more recent study reported an improved marginal fit for provisional crowns made from BisGMA composite resin provisional materials [8] using again the same methodology. Bis-GMA acryl resin composite provisional material resulted in $22 \mu \mathrm{m}$ of marginal opening, and a visible light cured provisional material $29 \mu \mathrm{m}$.

The difference in findings between our study and the previous studies might be explained by the fact that provisional restorations were placed on a water bath to complete polymerization at $37^{\circ} \mathrm{C}$ in an attempt to mimic body temperature but the mouth is often not subject to such high temperatures especially when open during treatment. It has been shown that curing of acrylic provisional materials in water baths significantly improves marginal fit of produced restorations [22].

In this investigation the materials were left to complete polymerization on room temperature which was significantly lower than $37^{\circ} \mathrm{C}$, which resulted in increased polymerization time and probably increased polymerization shrinkage. The clinically unrealistic use of the spring loaded device to secure the restorations over the analogue and the direct measuring of the marginal opening in just one axis might have also influenced the results due to compression of the provisional restorations over the analogue.

The differences in marginal discrepancies can also be the result of increased polymerization shrinkage of provisional restorations in our investigation as they were stored on dry air for one hour prior to the application of McLean and von Fraunhofer [24] technique. Polymeric materials exhibit continuous polymerization even after one week storage in dry air resulting in increased marginal discrepancies [23].

\section{Conclusions}

In implant supported restorations there is a need for long term provisional treatment compared over natural teeth. Longer provisional treatment time, longer spans, and the necessity for addressing tissue contour dictate the need for provisional materials with improved physical properties and good biocompatibility. The superiority of Bis-GMA acryl resin composite both in terms of physical properties and, from this experiment, the improved accuracy of fit compared to acrylic resin materials indicates that it may be the material of choice for fabrication of provisional restorations for implant abutments.

\section{References}

1. Burns DR, Beck DA, Nelson SK; Committee on Research in Fixed Prosthodontics of the Academy of Fixed Prosthodontics. A review of selected dental literature on contemporary provisional fixed prosthodontic treatment: report of the Committee on Research in 
Fixed Prosthodontics of the Academy of Fixed Prosthodontics. J Prosthet Dent. 2003; 90(5): 474-97.

2. Vahidi F. The provisional restoration. Dent Clin North Am. 1987; 31(3): 363-81.

3. Federick DR. The provisional fixed partial denture. J Prosthet Dent. 1975; 34(5): 520-26.

4. Higginbottom FL. Quality provisional restorations: a must for successful restorative dentistry. Compend Contin Educ Dent. 1995; 16(5): 442, 444-7.

5. Lowe RA. The art and science of provisionalization. Int J Periodontics Restorative Dent. 1987; 7(3): 64-73.

6. Zinner ID, Panno FV, Landa LS. Provisional Prosthodontic appliances in the combination of fixed-removable reconstruction. Dent Clin North Am. 1989; 33(3): 379-97.

7. Lui JL, Setcos JC, Phillips RW. Temporary restorations: a review. Oper Dent. 1986; 11(3): 103-10.

8. Tjan AH, Castelnuovo J, Shiotsu G. Marginal fidelity of crowns fabricated from six proprietary provisional materials. J Prosthet Dent. 1997; 77(5): 482-85.

9. Tjan AH, Tjan AH, Grant BE. Marginal accuracy of temporary composite crowns. J Prosthet Dent. 1987; 58(4): 417-21.

10. Young HM, Smith CT, Morton D. Comparative in vitro evaluation of two provisional restorative materials. J Prosthet Dent. 2001; 85(2): $129-32$

11. Moulding MB, Teplitsky PE. Intrapulpal temperature during direct fabrication of provisional restorations. Int J Prosthodont. 1990; 3(3): 299-304.

12. Wang RL, Moore BK, Goodacre CJ, Swartz ML, Andres CJ. A comparison of resins for fabricating provisional fixed restorations. Int I Prosthodont. 1989; 2(2): 173-84.

13. Fleisch L, Cleaton-Jones P, Forbes M, van Wyk J, Fat C. Pulpal response to a bis-acryl-plastic (Protemp) temporary crown and bridge material. J Oral Pathol. 1984; 13(6): 622-31.
14.Diaz-Arnold AM, Dunne JT, Jones AH. Microhardness of provisional fixed prosthodontic materials. J Prosthet Dent. 1999; 82(5): 525-28.

15. Koumjian JH, Nimmo A. Evaluation of fracture resistance of resins used for provisional restorations. J Prosthet Dent. 1990; 64(6): 65457.

16. Bohnenkamp DM, Garcia LT. Repair of bis-acryl provisional restorations using flowable composite resin. J Prosthet Dent. 2004; 92(5): 500-2

17.Zwetchkenbaum S, Weiner S, Dastane A, Vaidyanathan TK. Effects of relining on long-term marginal stability of provisional crowns. J Prosthet Dent. 1995; 73(6): 525-29.

18. Nejatidanesh F, Lotfi HR, Savabi O. Marginal accuracy of interim restorations fabricated from four interim autopolymerizing resins. Prosthet Dent. 2006; 95(5):364-67.

19. MouldingMB, Loney RW, Ritsco RG. Marginal accuracy of provisional restorations fabricated by different techniques. Int J Prosthodont. 1994; 7(5): 468-72.

20. Crispin BJ, Watson JF, Caputo AA. The marginal accuracy of treatment restorations: a comparative analysis. J Prosthet Dent. 1980; 44(3): 283-90.

21. Barghi N, Simmons EW. The marginal integrity of the temporary acrylic resin crown. J Prosthet Dent. 1976; 36(3): 274-77.

22. Ogawa T, Aizawa S, Tanaka M, Matsuya S, Hasegawa A, Koyano K. Effect of water temperature on the fit of provisional crown margins during polymerization. J Prosthet Dent. 1999; 82(6): 658-61.

23. Koumjian JH, Holmes JB. Marginal accuracy of provisional restorative materials. J Prosthet Dent. 1990; 63(6): 639-42.

24. McLean JW, von Fraunhofer JA. The estimation of cement film thickness by an in vivo technique. Br Dent J. 1971; 131(3): 107-11.

25. Bland J, Altman D. Statistic methods for assessing agreement between two methods of clinical measurement. Lancet 1986; 1(8476): 307-10. 